\title{
Expanding Applicability of Senior Projects: Portable EGCC for Greenhouses
}

Dr. Jorge Rodriguez P.E., Western Michigan University

Dr. Alamgir A. Choudhury, Western Michigan University

Alamgir A. Choudhury is an Associate Professor of Engineering Design, Manufacturing and Management Systems at Western Michigan University, Kalamazoo, Michigan. His MS and PhD are in mechanical engineering from NMSU (Las Cruces) and BS in mechanical engineering from BUET (Dhaka). His interest includes computer applications in curriculum, MCAE, mechanics, fluid power, and instrumentation \& control. He is a Registered Professional Engineer in the State of Ohio and affiliated with ASME, ASEE, SME and TAP. 


\section{Expanding Applicability of Senior Projects: Portable EGCC (Environmental Growth and Cloning Chamber) for Greenhouses}

Nowadays it has become a common practice to do senior design projects or capstone projects under the sponsorship of an industrial entity, or a technical business/agency, where students will be performing technical activities closely related to their specific engineering field. A wonderful opportunity for the students to realize the reach of their technical education is to participate in senior design projects where the connection to the field of study might not be that clear. This paper will present such situation, with the case of a portable cloning chamber.

A portable cloning chamber is basically a small clean room. This chamber is used in greenhouses for tissue culture and cloning of plant life. It is an enclosure that should remain sterile while being used to do plant cloning in a greenhouse environment. The chamber remains sterile through the use of laminar flow hoods and HEPA filters, thus controlling as well the flow of clean air through the chamber. Another component that help in keeping the level of sterility is ultraviolet lights, which kills existing bacteria thus allowing for a completely sterile environment.

The objective of this project was the design, build, and test a cloning chamber that has the versatility to serve in several stages of tissue culture. The chamber needs to maintain specific conditions in terms of air flow rate, temperature, humidity, cleanness, and lighting. Additionally, the chamber needs to be portable in order to be used in different rooms and at different locations in a greenhouse, low cost since most of the users are expected to be small businesses, and selfcontain so that there is no need for additional infrastructure.

This paper reports on the steps taken for the successful completion of the project by following a typical engineering design process, from the ideation stage to the implementation and testing stage. Particular emphasis given to conceptual engineering, i.e., define the problem, develop specifications, and brainstorming. The emphasis on these steps was in part due to the importance of doing background research in order for the team members to become well versed on the theory, use, and application of cloning chambers in greenhouses.

The project was a novel and challenging experience for all people involved, particularly for the students working on the project. It was an interesting experience for the small business sponsoring the project (expectations and requirements when sponsoring a senior project), students in the classroom (need to learn about the topic in order provide feedback and evaluate progress), and the faculty advisor (provide guidance in a non-typical technical topic). However, this project clearly showed the wide applicability of the engineering education that students have received during their coursework, and opened their eyes to a wider spectrum of possibilities.

\section{Background}

Capstone Projects. Whenever the terms senior design project and capstone project are used, the classical understanding will be for a project that is closely related to the field of study for the student(s), with the characteristic that the project will be as well closely related to the materials covered in the main courses in the program. This understanding has been changing in recent 
times. On one hand there is the tendency to present to students situations where they will be challenged by having to deal with a topic or application that is not usually understood as direct link to their classwork. On the other hand, there is the tendency to have more project sponsors from outside the academic environment. Both tendencies have been created with the intention of present real life situations to the students, and there have been growing pains, but such practices are gaining acceptance ${ }^{6}$.

The EDT program. The project was the capstone project for two students in the Engineering Design Technology (EDT) program. Senior design projects are a two-semester sequence where students bid on projects proposed by faculty members, and then students are assigned to teams. The projects can be sponsored or not, and external (off-campus) or not. Faculty member or members act as technical advisors for the project, and there is course coordinator for the class. The technical advisor is the liaison between the students and the sponsor(s). In recent past there has been an increase of request for projects that are not discipline specific, with small businesses exploring the option of a collaboration with college students. The team assigned to this project had only two students (typical team is 4 students, with 3 being the preferred number). Perhaps the novelty of the project, and the perceived challenge of being an unusual topic resulted in having fewer than the usual number of students.

Plant cloning. Contextual information for the project was one of the initial challenges for the team working on this project. The team had two students, both majoring in Engineering Design Technology, which is a program with emphasis on the design process with special focus on computational tools for analysis. Student had to dedicate additional time to learn about plant cloning ${ }^{2}$, which can be done in one of two ways, the first and most common method is the Cutting Method. This process involves simply cutting a branch - leaf and its stem - from the mother plant. Cut the plant 45 degrees from the cross section to increase surface area at the cutting. The cutting is dipped in a medium (paste) to provide nutrients to help the cutting develop roots, and due to the 45-degree cut helps the plant absorb the nutrients. The paste along with a constant supply of water allows the stem to grow its own root system, thus creating a clone of its parent plant. This method, though effective, is not without its flaws because only one clone can be created per leaf stem. Therefore, if a grower wants multiple clones the parent plant will have to be stripped of that many leaves and stems, and if the parent plant has contracted any diseases throughout its growth they will be transferred to the emerging clones.

A new system of plant cloning has recently been made available due to advancements in technology, and it has resulted in a reduction in starting cost. The second method is that of a tissue culture clone. A tissue clone can be taken from any segment of a host plant. It is then set in the nutrient medium to grow and can develop a root system within an agar medium. The method of plant tissue culture cloning allows for mass propagation, faster growth, and the ability to cut out any diseases that may have come into contact with the parent plant. In order to successfully harvest a tissue culture one must have a sterile environment to house them. The benefits to using the tissue culture method over the cutting method include:

- Asexual propagation

- Quickly produce mature plants

- Exact clones of specific plant

- Reproduce healthy clones from diseased plants to preserve its unique genes 
The negative aspects of tissue culture methods are:

- Requires a great degree of skill to keep everything clean and sterile

- Requires expensive equipment to properly regulate sterility of workstation.

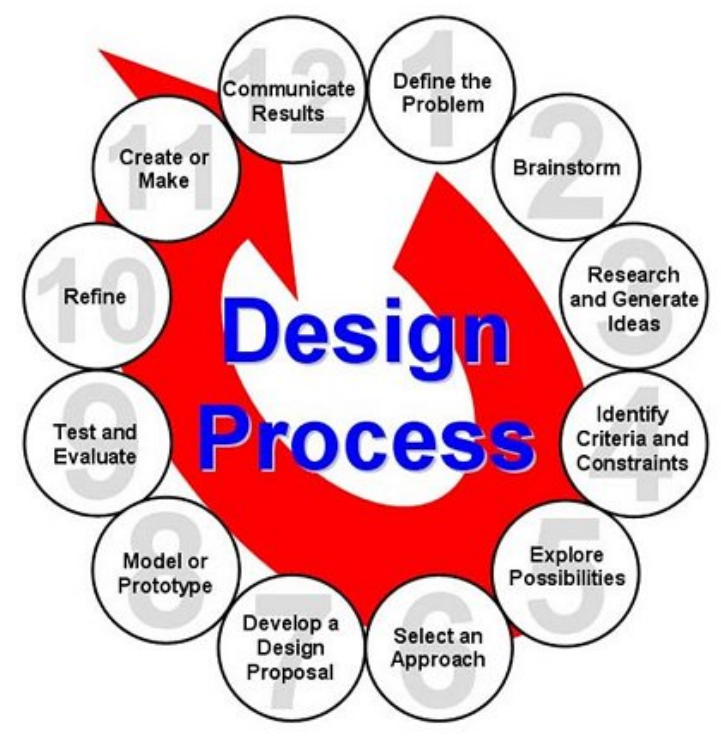

Figure1: Engineering Design Process ${ }^{11}$.

\section{Problem Statement}

Another challenge for most students is to come up with the proper problem statement for their project. The very first task assigned to each member of the team is to write the problem statement, objectives, and expected deliverables for the project. It is interesting to find out that the students that selected the project have significantly different problem statements. But it is a great exercise that serves as the basis to get everyone in the same page, and clarify the project ${ }^{5}$.

Demand for rare and exotic greenhouse plants has been on the rise recently, which has resulted in an increase in growth and maintenance costs. This situation has forced small greenhouses to find out how they can develop and take full advantage of their greenhouse capabilities, thus the need for a chamber that allows them to clone and grow plants in an optimal and sterile environment. This chamber with sterile environment is the focus of this project. The sterile environment, which will be dubbed 'cloning chamber' will have to not only be completely sterile and have the ability to stay that way during the entire cloning process, but also serve as housing chamber for multiple tissue cultures until they reach their transplanting stage. So, using the knowledge about design process, and applying the system integration skills that are provided in the EDT program, the team will design, build, and test a cloning chamber to the specifications provided by the sponsor.

The project objective can be stated as: to design and test an Environmental Growth and Cloning Chamber (EGCC) which would provide a sterile environment for the production of plant clones. The chamber should be lightweight, compact, and should not exceed $\$ 800$ in cost of materials.

\section{Methodology}


As indicated, the EDT program emphasizes understanding of the design process. A standard engineering design process was followed in this project (Figure 1). The typical conceptual, basic, and detail engineering phases have different steps. Some of the steps mentioned in the figure have already been established by virtue of presenting the project, but some other ones are described below.

\section{Brainstorm.}

Different concepts were proposed and combined in order to have a feasible solution to the problem. Key design components were listed in this step. Several drawing and sketches (Figure 2) were produced to help choose the best method to create a sterile chamber. HEPA filtration, UVGI sterilization and air straightening were considered here.
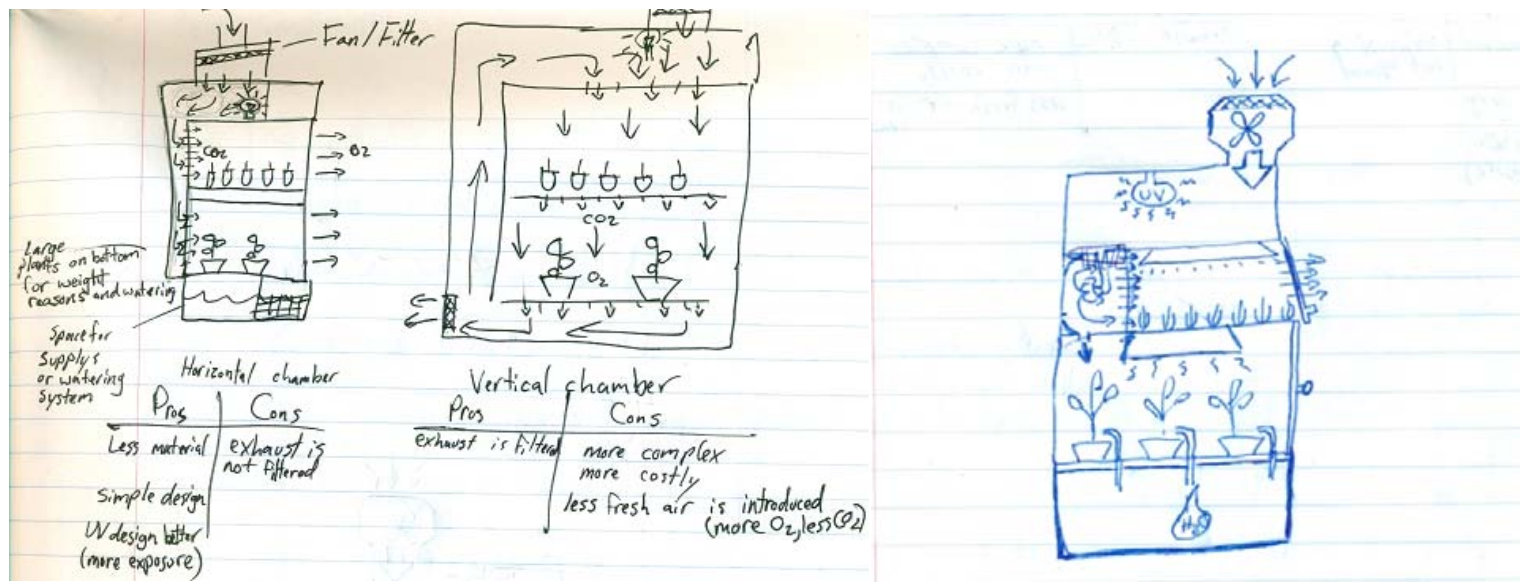

Figure 2: Sample of sketches from brainstorming sessions.

\section{Research.}

The main sources of information was staff at the sponsor's facilities. Visiting and speaking with them helped the students to get a good working knowledge of the context for the project. The students showed has well an interest on learning more about greenhouses, partially due to the fact that they were being asked many questions by their classmates, and the technical adviser. Standard information was gathered, thus learning that a typical greenhouse was crowded with different plants and equipment, the greenhouse is temperature and humidity controlled. Temperature remains at 70 degrees Fahrenheit and humidity is between 50-55\%. Most greenhouses have special lighting which can produce a significant amount of heat. It was interesting to observe how students were thinking about solutions as soon as they learned new facts - a great opportunity as well to emphasize issues like, documenting your project, need to be organized and follow the recommended steps in the design process flowchart. For example in this situation of temperature control and use of lamps, it was mentioned that one option is to use LED lights in order to keep the temperature down. The location where the EGCC will sit is a confined location with a maximum footprint of 28 inches wide and 42 inches deep, and leaving just enough room for the operator to stand in front of it.

Other technical topics that needed to be researched by the team in order to have a better understanding of the way cloning chambers function, and are utilized, are the following ones: Laminar Flow Hoods. The purpose of this type of hoods is to blow a constant laminar flow of filtered air across the workspace in the chamber in order to prevent contaminants from coming in 
contact with any element inside the chamber. The air is filtered by a High Efficiency Particulate Air (HEPA) filter. Laminar flow hoods are common in chemistry and biology labs. Laminar flow hoods can be divided into two categories, horizontal ${ }^{4}$ and vertical ${ }^{1}$.

HEPA filters. Its efficiency is directly related to the amount of air flowing through the filter, and some commercial HEPA filters will include recommendations for air flow ${ }^{3}$. Because such relationship, selection of HEPA filters and the blower should be done simultaneously. A prefilter is also used to help eliminate some of the larger particles from coming into contact with the HEPA filter. This will increase the time intervals between filter maintenance, and will increase the HEPA filters efficiency. Vertical flow hoods are primarily used when hazardous materials are being used in the chamber. Filtered air flows from top to bottom, and exhausts through a second filter to prevent any dangerous fumes from escaping from the chamber Horizontal flow hoods pump air from back to front, directly toward the operator, and using only one HEPA filter, making them simpler in design and lower in cost ${ }^{4}$.

Ultraviolet Germicidal Irradiation (UVGI). Ultraviolet (UV) light is a common method of destroying microorganisms. Ultraviolet radiation will alter the microorganisms DNA, leaving it without the ability to reproduce. Ultraviolet radiation is defined as "that portion of the electromagnetic spectrum described by wavelengths from 200 to $400 \mathrm{~nm}$ ”. . Exposure to UV radiation can be harmful to the operator only with prolonged exposure. UV-C radiation contains the wavelength ideal for this function, and it is defined within the ranging of 200 to $280 \mathrm{~nm}^{14}$. UVGI systems involve a bulb with a $265 \mathrm{~nm}$ wavelength installed in or near the source of the airflow but should be installed downstream from the filter, to prevent dust from dropping the bulbs efficiency. Another method to improve efficiency involves designing the area around the bulb with reflective surfaces allowing optimal air and surface disinfection ${ }^{9}$.

Basic Engineering. The next phase in the design process is to establish specifications for the equipment to be used in the proposed system. Once the students had a better understanding of cloning chambers and the way they operate, the basic engineering phase was more of a traditional set of calculations, closer to their engineering concepts. The following items were specified:

Material selection. Plywood with proper finish can be moisture and UV resistant as well as reflect light, which are all important aspects to the system. Plywood with mortise and tenon would eliminate the need for any extra framing. This would reduce material cost and weight. The doors were chosen to be without hinges because the swinging doors would not be able to open in the confined space where the chamber will be located in the greenhouse. Chamber material chosen: a) 3/8" plywood shell for low cost and weight, b) ultraviolet resistant interior paint, and c) staining finish for age, mold, and insect deterrent.

HEPA filter selection. Organic air HEPA filters are designed and created using opposing dual cone technology that optimizes airflow even as it filters out airborne particles, molds, insects and bacteria. These filters can be easily maintained, only needing a soft brush and water to clean both inside and out. In order to have this filter work efficiently it needs an airflow of 452-680 CFM (cubic feet per minute) to ensure a particle reduction of 91.34-91.50\% (See Table 1). Given a large fan with a variable speed controller we will be able to change wind speeds to suit our filter. Thus, a laminar flow chamber having both components ${ }^{3}$. 
Table 1: Example of specifications of a HEPA filter ${ }^{3}$

\begin{tabular}{|c|c|c|c|c|c|}
\hline \multicolumn{2}{|c|}{ Flow Rate } & Back Pressure & \multicolumn{3}{c|}{ Efficiency } \\
\hline CFM & $\mathbf{M}^{3} / \mathbf{H}$ & Pa & >1 micron & $\mathbf{> 2 ~ m i c r o n ~}$ & $\mathbf{3}$ micron \\
\hline 1132 & 1923 & 172.5 & 61.69 & $88.42 \%$ & $92.41 \%$ \\
\hline 904 & 1535 & 129.4 & 48.60 & $87.67 \%$ & $92.37 \%$ \\
\hline 680 & 1155 & 90.2 & 37.10 & $87.47 \%$ & $91.50 \%$ \\
\hline 452 & 767 & 51.90 & 26.19 & $78.73 \%$ & $91.34 \%$ \\
\hline
\end{tabular}

Fan selection. A 6” centrifugal fan was chosen for the EGCC. It creates a 435 CFM flow rate which will filter about $91 \%$ of any particles greater than 5 microns. Since most of the particles we are focusing on are greater than 10 microns, this fan will suffice.

Chamber design. The chamber was designed with two separate compartments. The top compartment was designed to provide a sterile place for the clones to develop. The top compartment included: a) HEPA filtered laminar air flow, b) air straightener ducting, c) UVGI lighting, and d) high efficiency UFO lighting. The bottom chamber was built to allow for growth of the plants after they have outgrown their test tubes. At that point the plants are no longer as susceptible to contamination, but require more lighting, watering, and space. The bottom compartment included: a) LED growth lighting, b) 24”x30”X24” workspace, and c) space for watering system and growth up to 12” high.

The system was also designed to allow the removal of the middle shelf, thus allowing for the entire chamber to be used to grow tall, matured plants. The air system can then be reversed, allowing to pull air (without filtration) to help reduce heat and increase air flow, both important factors to consider for larger plants.

Detail Engineering and Fabrication. These phases of the design process can be considered more traditional, resulting in fewer additional requirements from the students. Team members were more in their comfort zone because this phases is something that they did perform during regular coursework.

$C A D$ model. A solid model was generated to help in the visualization of the complete proposed system. The ducts were designed during the creation of the CAD model utilizing routing techniques covered in coursework. Having a CAD model helped as well to provide a better sense of size of the system, and proper locations for the components. Once the complete CAD model was created as an assembly, standard documentation of the design was produced for presentation purposes. The chamber was built based on our finalized model (Figure 3). 

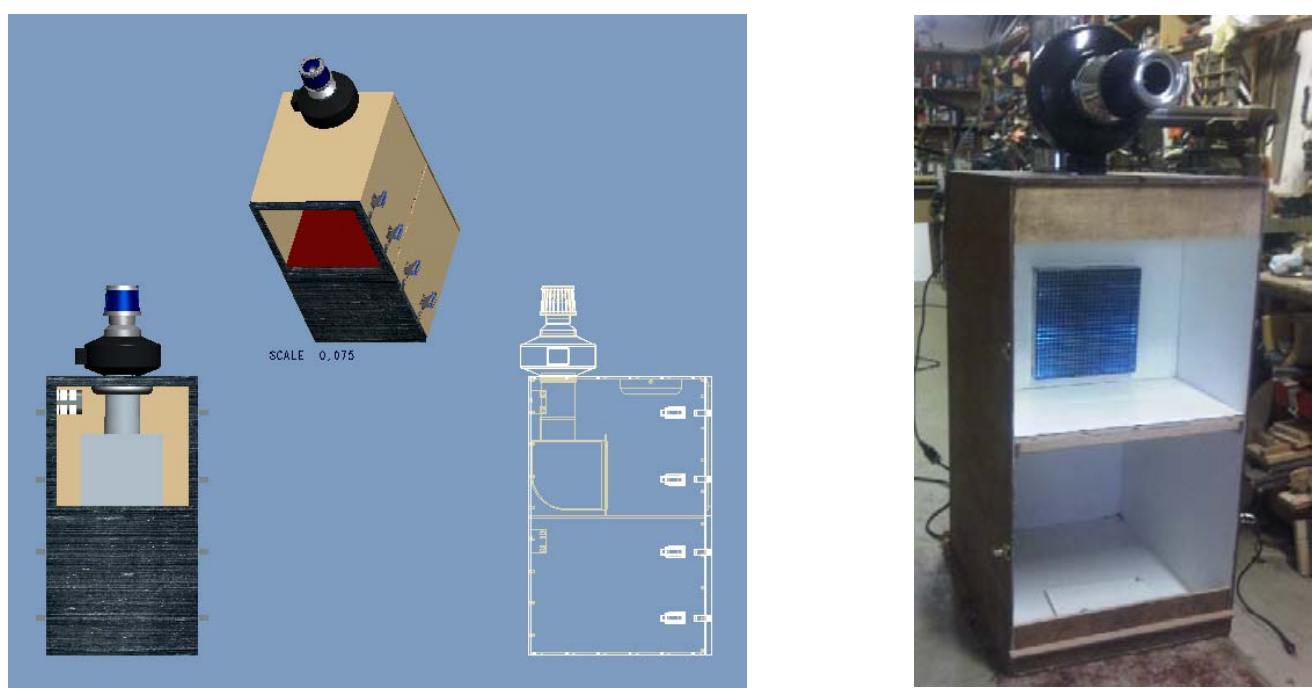

Figure 3: CAD model for proposed design, and picture of final product.

Assembly and Testing. The chamber was built using a mortise and tenon assembly technique, thus creating a sturdy box with no nails or screws. The shelf is removable to allow G\&S Landscaping to use the chamber as they see fit. The doors could be open and closed by one person incorporating a latching system. The interior of the chamber was painted with white reflective paint that was resistant to mold, moisture, and UV lighting. The outside of the chamber was finished with a wood sealant that was resistant to mold, moisture, aging, and insects. The ducting was pieced together using galvanized aluminum and sealed with plastic grommets and sealants to ensure no air leaks both inside and out. The electrical work was assembled with remote operated features to reduce human contact and therefore reduce the risk of contamination.

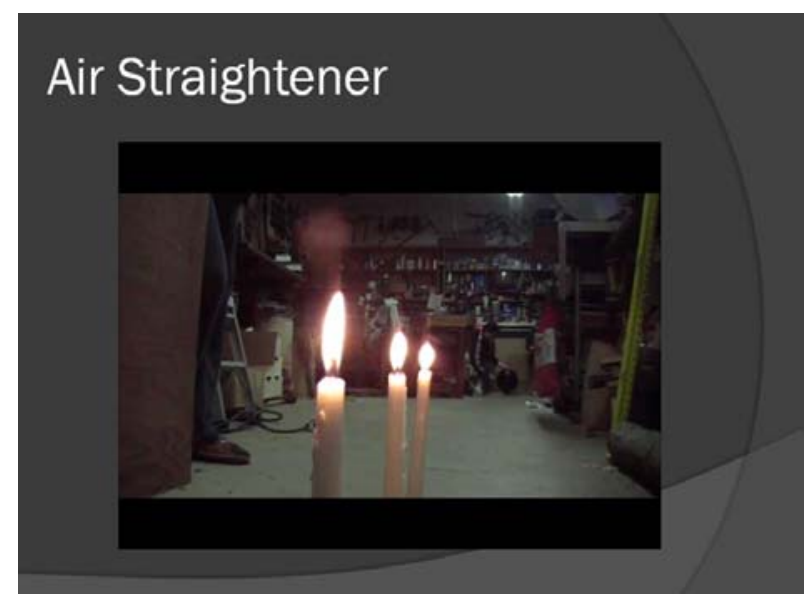

Figure 4: Testing of the air straightener system.

Testing was done on the air flow exhausting from the chamber to assure that the air straightener specified for producing laminar flow were working as intended ${ }^{13}$. The test was observationbased: if the flame in several candles will not be disturbed as there is airflow, then the air flow is as expected (Figure 4). It was found that the air straightener helped project the air by an average of 12 feet, which is adequate for the proposed design. 
Cost Comparison. The EGCC was built to be quite universal incorporating a cloning chamber and a two stage growing chamber. This feature is something that cannot be found in commercial chambers, it implies two different chambers. The materials had a total cost of $\$ 731$, which is within the allocated budget, and compares very well with a commercial chamber costing just under $\$ 3,000$. Couple of comments are that some for the components used to build the EGCC were low-balled for cost estimation by assuming purchase of sets or in large quantities, and labor cost is not included.

\section{$\underline{\text { Evaluation }}$}

This senior design project presented an opportunity to a team of students to get outside their comfort zone and face a situation where, even when the technical concepts applied are the ones that were presented to them during their coursework, additional research needed to be performed in order to properly address the problem at hand. The experiences provided by this type of capstone project are in line with the trends and expectations that have been previously reported in the literature ${ }^{10}$. There was a component that presented a real problem, and there is a component where soft skills were developed ${ }^{12}$. The project was an open-ended problem where team members had to think about safety and functionality, and at the same time team members developed and applied skills on teamwork, project planning, economics, and communication ${ }^{7}$. The key difference was the domain where the students had to develop their project, the project was not a typical application of what they have learned in their coursework, but at the same time this project presented the opportunity to illustrate a common situation that students will be faced once they apply (and get) a technical job. It brings to light one of the message that are usually offered in design courses where open-ended and different application domains are utilized. These situations represent a challenge to students and faculty because there are more requirements on their time; but at the same it represents an opportunity to illustrate the applicability of engineering in a wide-variety of fields. As indicated, everyone involved in such projects benefit from the experience, and should be encouraged.

\section{Conclusions}

Technically the project was a successful one. The cloning chamber fulfilled and exceeded all project objectives given by the sponsor, and all the components and functions were operational. The EGCC has been in operation for few months now, and there has not been any report of major issues. There has been comments related to preferences or improvements (e.g., ergonomics - adjustable height of removable shelf). Another comments received by the sponsor for this report is that it has performed well, with the only issue being the care they have to give to the system whenever they are moving it from one greenhouse to another. So, a more robust built is suggested in case a new unit is built.

Similarly, the project was a learning experience for all parties involved. Team members had to learn about topics that are not typically covered in classes, as well as get well familiarized with the topic of greenhouses, so that the decision process was based on correct assumptions, and the important factors are given proper emphasis. There were occasions at the start of the project when an idea/concept was discussed with the sponsor, just to find out that there were important factors that the team was missing and not considering. A great learning experience, without a 
doubt. The technical adviser benefited from the experience as well because the project gives a window of opportunity to showcase the universality of the technical concepts covered in the classroom. And finally, the project benefited the sponsor by getting a needed piece of equipment at an affordable price.

In conclusion, this type of projects are a win-win-win situation that do lend themselves for many other uses in academics. Students have the satisfaction of producing results in a more challenging situation, even the faculty member proposing the project was not sure if the project will generate enough interest and students will bid on it. Most students nowadays are really taxed with all the activities they need to do in order to attend college. The percentage of students that are considered full-time students is dropping year after year, perhaps more so in the engineering technology programs. Fortunately the project was selected and it had good results.

\section{Acknowledgements}

This project was sponsored by G\&S Landscaping. Greg Amaro and Joseph Simko were directly involved with the project, and the technical staff at the machine shop provided valuable support during the building and testing phases.

\section{References}

1. Air Clean Systems. (2008). Vertical Laminar Flow Workstation. Retrieved 12 8, 2010, from Laminar Flow Hoods: http://www.aircleansystems.com/process_vertical.htm

2. Amaro, G. (2010, October 17). Plant tissue culture information meeting. (J. S. Greg Amaro, Interviewer)

3. Deau Passe. (2010). 4" HEPA Filters. Retrieved November 12, 2010, from Organic Air Filters: http://www.organicairfilter.com/product_detail_Hepa4inch.shtml

4. Favero, M. S., \& Berquist, K. R. (1968). Use of Laminar Air-Flow Equipment in Microbiology. Applied Microbiology, Vol. 16 No. 1, 182-183.

5. Gorman, M. F. (2010), The University of Dayton operations management capstone course: Undergraduate student field consulting applies theory to practice. Interfaces, vol 40, no. 6, pp. 432-443.

6. Jassim, E. (2014), Formulation of Capstone Design Projects for Experiential Learning, Proceedings of Capstone Design Conference, Columbus, $\mathrm{OH}$.

7. Kanai, J. and Anderson, Mark (2012), Helpful Guidelibnes in Working with Industry Sponsors, Proceedings of Capstone Design Conference, Champaign-Urbana, IL.

8. Key, M. M. (1972). Occupational exposure to ultraviolet radiation. U.S. Department of Health, Education, and Welfare.

9. Martin, S., Dunn, C., \& Freihaut, J. (2008). Ultraviolet Germicidal Irradiation Current Best Practices. American Society of Heating, Refrigeration and Air-Conditioning Engineers, inc, 28-36.

10. Pembridge, J.J. and Paretti, M.C. (2010) The current state of capstone design pedagogy. Proceedings of ASEE Annual Conference and Exhibition, Lousville, KY.

11. Project Lead the Way (PLTW) Igniting imagination and innovation through learning (2013). Unit 1 Lesson 2 Documenting Process.

12. Shuman, L. J., Besterfield-Sacre, M., and McGourty, J. (2005). The ABET professional skills - Can they be taught? Can they be assessed?, Journal of Engineering Education, vol. 94, no. 1, pp. 41-55.

13. Sunleaves Garden Products. (2010). Sunleaves WindTunnel (SW608). Retrieved December 8, 2010, from sunleaves.com: http://sunleaves.com/detail.asp?sku=SW608

14. Ultraviolet Devices Inc. (2009). UVDI: How It Works. Retrieved Nov. 23, 2010, from UVDI.com; http://uvdi.com/pages/how-it-works. 